\title{
Analyzing the effect of clock jitter on Self-Oscillating Sigma Delta Modulators
}

Dries Vercaemer, Pieter Rombouts

This document is an author's draft version submitted for publication to IEEE Trans. Circuits Syst.-I: Regular Papers.

The actual version was published as [1].

\section{REFERENCES}

[1] D. Vercaemer and P. Rombouts, “Analyzing the Effect of Clock Jitter on Self-Oscillating Sigma Delta Modulators," IEEE Trans. Circuits Syst.-I: Regular Papers, vol. 63, no. 2, pp. 200-210, FEB 2016. 


\title{
Analyzing the effect of clock jitter on Self-Oscillating Sigma Delta Modulators
}

\author{
Dries Vercaemer, Pieter Rombouts
}

\begin{abstract}
This paper presents simple but accurate expressions for the noise components caused by clock jitter, in the output signal of Self-Oscillating Sigma Delta Modulators (SOSDMs). Contrary to conventional Continuous Time Sigma Delta Modulators (CTSDM), the SOSDM's loop contains a strong oscillation, whose attribution to the system's jitter caused noise has not previously been explored. In this paper, the SOSDM system is modeled, and the effect of the self oscillation, the input signal and the quantization noise on the jitter caused noise in the output signal, is calculated. Results are confirmed by system level simulations.
\end{abstract}

Index Terms-Analog-to-digital conversion, limit cycle, Timeencoding, jitter, self-oscillating sigma delta modulator, pulsewidth modulation.

\section{INTRODUCTION}

$\mathbf{S}$ EVERAL papers have been published ([1]-[4]) on the effect of clock jitter on the performance of continuous time sigma delta modulators (CTSDM). Most have considered the jitter to be white noise, although for real world jitter this is never the case. A clock generated by a phase locked loop (PLL) for instance, has a more complex phase noise spectrum, containing contributions from several noise sources, each of them with a different spectral shape. This is important, because the shape of the jitter spectrum has a significant influence on the total amount of in-band jitter-caused noise at the output of the modulator, even if the total clock jitter power remains unchanged. This was already shown in several more recent papers, which did include the effect of the jitter spectrum $([5,6])$ on the CTSDM's performance.

In this paper, we will expand these previous results by analyzing the effect of none-white jitter on self-oscillating sigma delta modulators (SOSDMs [7]-[12]). Unlike CTSDMs, the SOSDM's loop contains a strong high frequency oscillation. We will show that this oscillation will be modulated by clock jitter, leading to a noise component in the SOSDM's output signal. This noise component can not be neglected, and it has not previously been studied.

The rest of this paper is organized as follows: Section II describes and models the SOSDM system. Section III explores the effect of jitter on the system, models it, and offers the general equations. Based on this, it will be shown that the

Manuscript received $\mathrm{XX} \mathrm{XX}, \mathrm{XXXX}$; revised $\mathrm{XX} \mathrm{XX}, \mathrm{XXXX}$. This work was supported by the Fund for Scientific Research Flanders (FWOVlaanderen), Belgium.

D. Vercaemer and P. Rombouts are with the Department of Electronics and Information Systems(ELIS), Ghent University, 9000 Gent, Belgium (e-mail: dvercaem@elis.ugent.be; pieter.rombouts@elis.ugent.be)

Copyright (c) 2015 IEEE. Personal use of this material is permitted. However, permission to use this material for any other purposes must be obtained from the IEEE by sending an email to pubs-permissions@ieee.org.

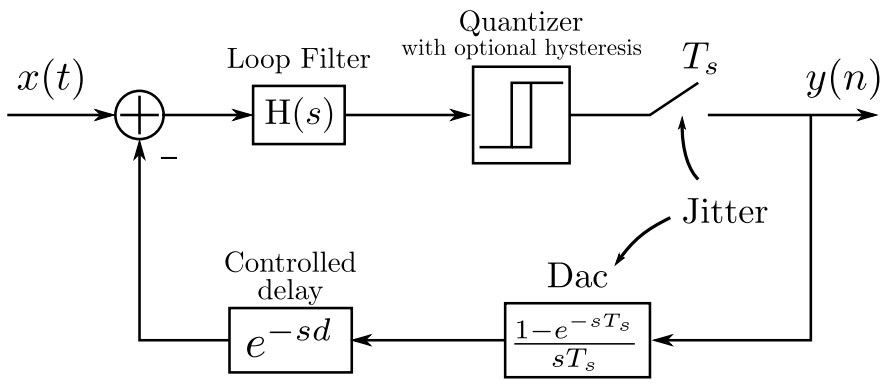

Fig. 1. A self-oscillating sigma-delta modulator

noise component due to jitter in the SOSDM's output signal, which we will call jitter noise for brevity, consists of three distinct components. This is a clear contrast with respect to the CTSDM, whose output signal consists of only two jitter related noise components. In section IV, we discuss the SOSDM's jitter noise components, and offer exact analytical expressions for them. We also derive simplified approximate expressions for the practically important case of clock signals whose jitter spectra exhibit first order roll off. Section V reports several system level simulation results that confirm the analytical expressions. In section VI, we offer some qualitative insights into the effect of spurs in the jitter spectrum on the SOSDM's performance, after which conclusions are drawn in section VII.

\section{Self-Oscillating Sigma Delta Modulators}

\section{A. The SOSDM's operation and properties}

Fig. 1 shows a conceptual diagram of a self-oscillating sigma delta modulator. The system is very similar in appearance to a conventional single bit continuous time sigma delta modulator. Just like a single bit CTSDM, it consists of a feedback loop with a loop filter, a one bit quantizer, a sampler and a digital to analog converter (DAC). However, its behavior differs strongly from that of a conventional continuous time sigma delta modulator, because the SOSDM's loop is forced to oscillate at a controlled frequency, either by inserting a well defined explicit delay in the feedback loop, or by using a Schmitt trigger, with a suitable hysteresis, as a quantizer. The oscillation frequency $f_{c}$, which should be much higher than the baseband frequencies, will then be a function of the delay, and/or of the hysteresis.

Introducing an oscillation into the modulator's loop could seem counter-intuitive to CTSDM designers, since the presence of an oscillation in CTSDMs is usually considered as detrimental for performance. It has however been shown in previous publications $([7,11,13])$ that introducing a controlled 
oscillation does not necessarily render the modulator useless, but instead can offer some attractive properties. Firstly, the SOSDM's loop operates at the oscillation frequency instead of the sampling frequency, (which is always significantly higher). This means that its power consumption can be reduced. But at the same time, for noise shaping purposes, the SOSDM still makes use of the much higher sampling frequency. Hence its performance is better than that of an equivalent single bit CTSDM sampled at the oscillation frequency. Secondly, because an explicit delay has been introduced, the SOSDM tends to be more robust against extra delays resulting from parasitic capacitors or process variations. Lastly, the frequency of operation of the SOSDM's loop is an extra design parameter that offers the designer more flexibility in searching a modulator that is tailor made for a certain application.

Several researchers have noticed these advantages, and severall SOSDM designs have been published $([7,10,12,13])$. However, despite this interest, the SOSDM system still remains a research topic, and several aspects of the SOSDM have not sufficiently been analyzed. In this paper, we will focus on the jitter behavior of the SOSDM system, and we will offer analytical results that can help designers in predicting the jitter performance of their systems.

In order not to overcomplicate the analysis, we will base the discussion in this paper on the case of a SOSDM architecture with an explicit delay $d$ in the loop and without hysteresis in the quantizer. Most of the following analysis however remains valid for a SOSDM with hysteresis. Although the actual expressions for the oscillation frequency and the linearized quantizer gain differ, the jitter mechanism remains unchanged, and the equations derived in sections III and IV are identical for both the architectures with and without quantizer hysteresis.

For notational convenience, we choose the quantizer's output voltage to switch between +1 and -1 . We will also restrict ourselves to DACs with non-return-to-zero (NRZ) pulses, which is by far the most common case.

Before we analyze the jitter behavior of the SOSDM, we will first model the system, and determine its output signal. In order to do so, we will build a linear model for the quantizer. However, because of the strong oscillation present in the SOSDM's loop, a straightforward linearization of the quantizer will not provide an accurate model. But if we think of all signals in the system of Fig. 1 as the superposition of this high frequency oscillating signal and a low frequency component, we will be able to linearize the comparator with respect to this low frequency component, even if it acts non-linearly on the oscillation. This approach is similar to established dual input describing function techniques [14].

We model the oscillation first, after which we linearize the comparator to determine the low frequency components at the SOSDM's output.

\section{B. Modelling the self-oscillation}

In order to model the oscillation, we study the simplified system of Fig. 2. In this system, we assume the input signal $x$ to vary slowly with respect to the oscillation, such that we

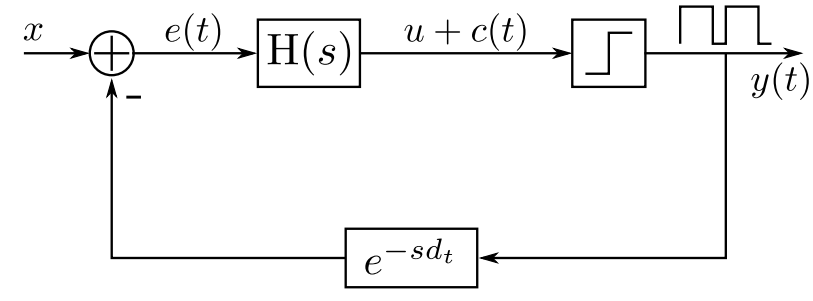

Fig. 2. The SOSDM with the sampling neglected.

can approximate it as a constant. We also neglect the sampling, but retain the half clock cycle delay of the NRZ pulse, which leads to a total loop delay equal to $d_{t}$ :

$$
d_{t}=d+\frac{T_{s}}{2}
$$

Here, $T_{s}$ represents the sampling period. By neglecting the sampling, the system of Fig. 2 actually represents an asynchronous sigma delta modulator (ASDM, [15]-[18]). The ASDM's output signal has been analyzed in literature and its oscillation frequency, $f_{c}$, has been calculated ([17]), for slowly varying input signals. It takes the following form:

$$
f_{c}=\left(1-x^{2}\right) f_{0}
$$

Here, $f_{0}$ represents the oscillation frequency of the ASDM if its input signal is zero. In the appendix, an expression for $f_{0}$ is given [Eq. (49)] which was derived in [15] for general order ASDMs (be it under certain assumptions on the loop filter). Later in this paper we will also derive an expression for $f_{0}$ for a first order loop filter [Eq. (10)], which, as we will argue, can be used as an approximation for higher order loop filters.

To facilitate the analysis of the jitter noise, we will approximate the square wave oscillation at the modulator's output, $y_{c}$, by its first Fourier component, a sine wave with amplitude $A_{c}$ and angular frequency $\omega_{c}$ :

$$
y_{c}(t)=A_{c} \sin \left(\omega_{c} t\right)
$$

As indicated by Eq. (2), and as has been shown in [17], the oscillation signal is modulated by the ASDM's input signal. Furthermore, in an actual realization, the oscillation signal may also contain phase noise caused by several noise sources in the system. As a result, both $A_{c}$ and $\omega_{c}$ will vary (relatively slowly) with time, and the first Fourier component of the oscillation will have sidebands. However, for the jitter analysis in this paper, we will neglect the phase noise. We will also remove the side bands by calculating the average oscillation amplitude and frequency, and using them to represent the oscillation as a single tone. In order to do this, we assume this input signal, $x(t)$, to be a sinusoid with amplitude $A_{x}$ and angular frequency $\omega_{x}$ :

$$
x(t)=A_{x} \sin \left(\omega_{x} t\right) \text { and } \omega_{x} \ll \omega_{c}
$$

For this input signal, the average value of Eq. (2) is readily derived:

$$
f_{c}=f_{0}\left(1-\frac{A_{x}^{2}}{2}\right)
$$


In the appendix, the average value of the oscillation's amplitude is calculated, starting from the theory of [15], with the following result:

$$
A_{c}=\frac{4}{\pi} \frac{1}{\sqrt{2}} \sqrt{1+\mathrm{J}_{0}\left(A_{x} \pi\right)}
$$

Here $\mathrm{J}_{0}$ is the zeroth-order Bessel function of the first kind. Eqs. (5) and (6) show that increasing the input signal amplitude will shift the oscillation's spectrum to lower frequencies, and will lower the amplitude of the fundamental tone.

In deriving the above results, we neglected the sampling operation. Although the resulting expressions for the frequency and amplitude of the oscillation signal will not be identical to those of a sampled system, we will use them as an approximation. Since the oscillation frequency is significantly lower than the sampling frequency, we expect these expressions to be quite accurate for SOSDMs.

To validate the analysis in this paper, we designed two third order SOSDMs, and used them to perform several system level simulations. The loop filters of both modulators have the same form, shown in Table I. The loop filter coefficients and system parameters of the modulators are listed in Table II. As can be seen, the first modulator has a delay of one clock cycle and an OSR of 50, while the second modulator has a delay of 2.5 clock cycles, and double the OSR. The integrator coefficients were obtained through brute force numerical optimization for high quantization noise suppression and good robustness.

To simulate the designed modulators, we built a behavioral model similar to the system depicted in Fig. 1. It incorporates a two level quantizer and a sample and hold block. For jitter simulations, this block samples at stochastically varying time instances, so that all jitter effects are modeled.

The results of a first set of simulations can be seen in Fig. 3. Here, the modulators were driven by a sinusoidal input signal with frequency $f_{x}$. For the first modulator, $f_{x}$ equaled $\frac{f_{s}}{200}$, while the input signal frequency of the second modulator was $\frac{f_{s}}{400}$. The simulations were performed for several values of the input signal amplitude, $A_{x}$. For every value, the average oscillation period was calculated. The results are plotted together with the analytical prediction of Eq. (5), using Eq. (49) to calculate $f_{0}$. Based on the plot, we can conclude that the analytical and simulation results show reasonable correspondence.

When the input amplitude becomes too large (i.e. roughly 70 percent of full scale), the systems shifted to another undesired mode of oscillation with a far lower oscillation frequency. Hence, the oscillation amplitudes at the internal nodes of the systems went far higher than full scale. This phenomenon is similar to instability due to overloading in a conventional CTSDM. It is a well known phenomenon in higher order CTSDMs, and the fact that the input signal level of a SOSDM should also be limited to a value lower than full scale comes as no surprise.

\section{Linearized modeling}

In order to obtain analytical results, and to understand the effect of jitter on the modulator, we need a linear model for

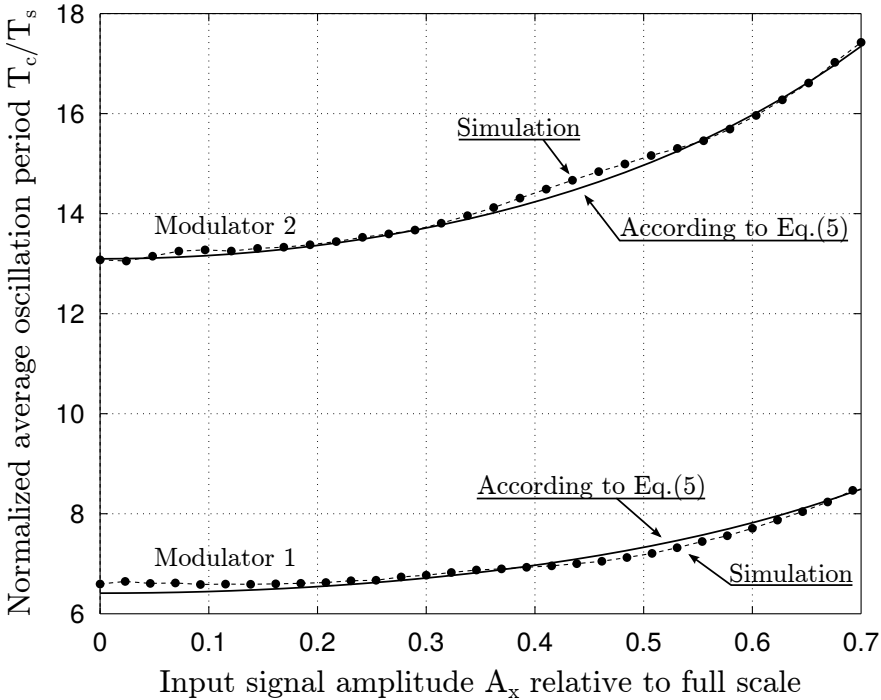

Fig. 3. Simulated averaged oscillation periods of two third order SOSDMs (modulators 1 and 2 in Table II), simulated for several input signal amplitudes. Analytical results are also plotted.

TABLE I

LOOP FILTER OF THE TWO THIRD ORDER SOSDMS USED IN THE SIMULATIONS. FIG. 1 SHOWS THE ARCHITECTURE.

$$
\begin{array}{|l|l|}
\hline H(s)=\frac{c_{3}}{s} \frac{s^{2}+c_{1} s+c_{1} c_{2}(1+g)}{s^{2}+c_{1} c_{2} g} & g=\frac{(0.78 \pi \mathrm{OSR})^{2}}{c_{1} c_{2}} \\
\hline
\end{array}
$$

TABLE II

PARAMETERS OF THE TWO THIRD ORDER SOSDMS USED IN THE SIMULATIONS. TABEL I SHOWS THE LOOP FILTER.

\begin{tabular}{|l|c|c|c|c|c|}
\hline & $c 1 T_{s}$ & $c 2 T_{s}$ & $c 3 T_{s}$ & $d f_{s}$ & OSR \\
\hline Modulator 1 & $0.025 \pi$ & $0.1 \pi$ & $0.05 \pi$ & 1 & 50 \\
\hline Modulator 2 & $0.004 \pi$ & $0.0098 \pi$ & $0.0225 \pi$ & 2.5 & 100 \\
\hline
\end{tabular}

the quantizer. To obtain this linear model for low frequency signal components, we initially assume that the input signal to the modulator, $x$, is a constant. We also assume that the loop filter of the system of Fig. 2 can be approximated by a first order filter: $H(s)=\frac{1}{s \tau}$. Practical systems may use higher order loop filters. However, these filters are often designed to have approximately first order roll-off at high frequency ([7]$[10,12,13])$, such that even for such high-order systems, the following analysis remains approximately valid.

The waveforms of the simplified system are depicted in Fig. 4 for a positive input signal $x$. It shows the output signal of the modulator, $y(t)$, the error signal in front of the loop filter, $e(t)$, and the triangular waveform at the comparator's input. The latter is represented as the superposition of a constant, $u$, and a zero-mean saw-tooth, $c(t)$. It is known that applying the superposition of a zero-mean saw-tooth and a constant or slowly varying signal to a comparator, leads to a square wave signal at its output, whose duty cycle is a linear function of this constant signal ([19]). This mechanism is called pulse width modulation. We also represent this pulse width modulated square wave as the sum of a zero-mean oscillation signal $y_{c}(t)$, and a constant signal $y_{x}$. The mean value of a square wave 


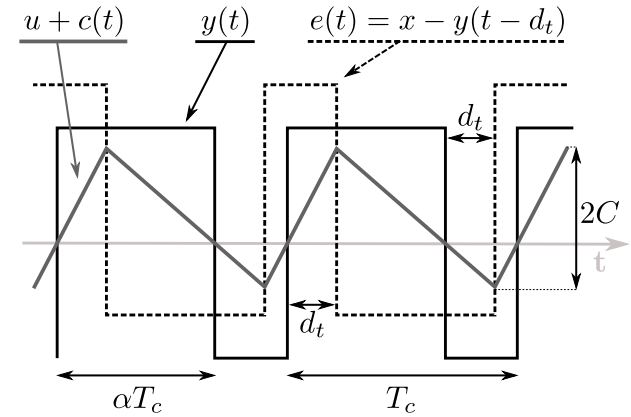

Fig. 4. Waveforms at several points in the first order ASDM, for a constant and positive input signal $x$.

with duty cycle $\alpha$, is given by:

$$
y_{x}=2 \alpha-1
$$

Keeping in mind that the loop filter is an integrator with time constant $\tau$, the rising and falling slopes of $\mathrm{c}(\mathrm{t})$, respectively $s_{r}$ and $s_{f}$, are found to be:

$$
s_{r}=\frac{x+1}{\tau}, s_{f}=\frac{x-1}{\tau}
$$

Using this result, and by examining the waveforms of Fig. 4, the following expressions are derived:

$$
\begin{aligned}
C & =\frac{d_{t}}{\tau} \\
f_{0} & =\frac{1}{4 d_{t}} \\
u & =C y_{x}
\end{aligned}
$$

Where $C$ represents the amplitude of $c(t)$, the oscillation in front of the comparator. From the last equation, the comparator gain for low frequency signals is evident:

$$
G_{u}=\frac{1}{C}=\frac{\tau}{d_{t}}
$$

Not surprisingly, this gain depends on the oscillation's amplitude in front of the comparator, as the oscillation acts as a carrier for the underlying PWM mechanism. Now it is also clear why this gain remains accurate for higher order SOSDMs, as long as their loop filters behave approximately as first order filters for the frequencies starting from $f_{c}$. For such filters, $c(t)$ will still resemble the saw-tooth of Fig. 4, and thus the comparator gain will approximate Eq. (12), and the PWM mechanism remains linear in good approximation.

For this linearization, we assumed the input signal to be a constant. The previous analysis however, remains approximately valid if the input signal varies slowly with respect to the oscillation. We now use the derived expression for the comparator gain to build a linear model for the low frequency components in the loop. As can be seen on Fig. 5, this model contains a noise source, $q(n)$, that is related to sampling. Until now, sampling was neglected, but because the information about $x(t)$ is coded as the width of a pulse (i.e. in the time domain), sampling (i.e. a quantization in time) leads to loss of information. This effect was studied in [7] where it was shown

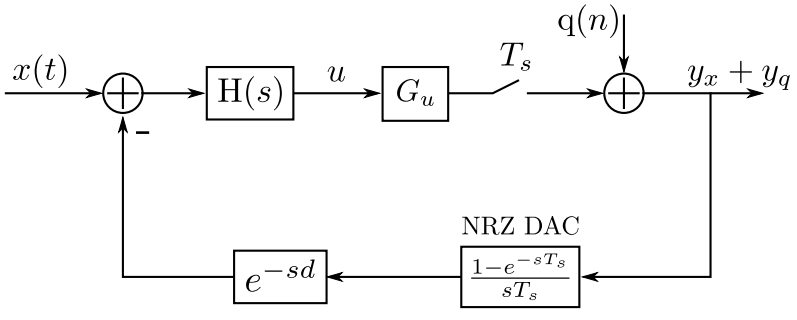

Fig. 5. Linear model for the input signal and the quantization noise

that it can be modeled as white additive Gaussian noise with a variance of $\sigma_{q}^{2}$ :

$$
\sigma_{q}^{2}=\frac{2}{3} \frac{f_{c}}{f_{s}}
$$

Here $f_{s}$ stands for the sampling frequency. We will use the system of Fig. 5 to determine $y_{q}$, the component in the overall output signal related to the quantization noise. We expect that the derived expression will at least be accurate for the low frequency components in $y_{q}$.

\section{Combined output}

We conclude that the output signal contains three components; an input signal related component, a quantization noise related component, and an oscillation component or carrier. The presence of this last component is the main difference between a conventional CTSDM and the SOSDM system. Using the linearized model for the non-oscillating components, we are now able to offer expressions for all SOSDM components in the Z-domain:

$$
\begin{aligned}
Y(z) & =Y_{x}(z)+Y_{q}(z)+Y_{c}(z) \\
& =\{X(s) \operatorname{STF}(s)\}^{*}+Q(z) \operatorname{NTF}(z)+Y_{c}(z)
\end{aligned}
$$

$\operatorname{STF}(\mathrm{s})$ and $\operatorname{NTF}(\mathrm{z})$ are the $\mathrm{S}$ - and Z-domain signal and noise transfer functions of the modulator:

$$
\begin{aligned}
\operatorname{NTF}(z) & =\frac{1}{1+\operatorname{LG}(z)} \\
\operatorname{STF}(s) & =\frac{G_{u} H(s)}{1+\left.\operatorname{LG}(z)\right|_{z=e^{s T_{s}}}} \approx 1 \\
\operatorname{LG}(z) & =\left\{G_{u} H(s) \operatorname{DAC}(s) e^{-s d}\right\}^{*}
\end{aligned}
$$

$\{A(s)\}^{*}$ represents the star operator operating on $\mathrm{A}(\mathrm{s})$ [20]. It is a short notation for taking the Z-transform of the sampled inverse Laplace transform of $A(s)$. LG $(z)$ is the Z-domain loop gain of the modulator. In the following analysis, the signal transfer function will always be assumed to equal unity for simplicity. This is a good approximation in all practically relevant cases.

To illustrate the spectrum of the output signal of a SOSDM, a simulation result for the first modulator in Table II is shown in Fig 6. This simulation was performed for an input signal amplitude of $-10 \mathrm{dBfs}$, and an input signal frequency equaling $\frac{f_{s}}{200}$. This resulted in a signal to quantization noise ratio of $66.7 \mathrm{~dB}$, which corresponds well with the analytically predicted value of $67.3 \mathrm{~dB}$. The average oscillation frequency obtained through simulation equaled $\frac{f_{s}}{6.85}$, while Eq. (5) predicted a 


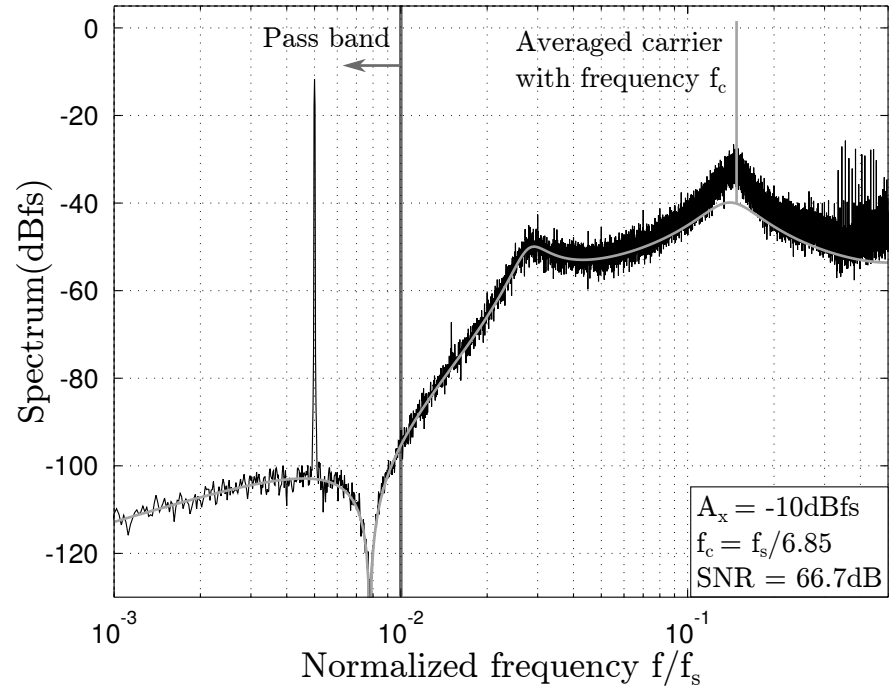

Fig. 6. Simulated and analytically obtained output signal spectrum of a SOSDM (modulator 1 in Table II) without jitter (obtained through averaging 10 periodograms of 10000 points).

value of $\frac{f_{s}}{6.75}$, using Eq. (49) for $f_{0}$. On Fig. 6, the linear approximation of the shaped quantization noise, and the sinusoidal approximation of the oscillation are also plotted. Fig. 6 shows excellent correspondence between simulation and analytical results for frequencies almost up to the oscillation frequency. At higher frequencies the curves deviate. This is to be expected since the analytical model of Eq. (12) is a low frequency approximation. The plot also illustrates that the oscillation has a broad spectrum, because it is modulated by the input signal and by the quantization noise. However, for most of the clock jitter spectra considered in this paper, approximating the oscillation as a sinusoid yields accurate results for the jitter noise, as the simulation results will show. Furthermore, it greatly simplifies the analysis, and yields simple closed form expressions.

There is however one situation where approximating the oscillation as a single tone introduces significant errors. If the jitter noise spectrum contains spurs around the oscillation frequency, a more accurate model should be used to describe the oscillation.

Such spurs could, for example, be present if the clock signal is generated by a PLL with a fractional divider [21]. The jitter noise of a SOSDM driven by a clock whose jitter spectrum contains spurs is not the main focus of this manuscript, but some simulation results will be given later in this paper, illustrating that these spurs can have a severe effect on the modulator's performance.

\section{MODELING THE EFFECT OF JITTER}

\section{A. Clock jitter}

Before we calculate the effect of jitter on the SOSDM's performance, we will first model the jitter as it would occur in a real-life system. An ideal clock has rising edges at every time instant $n T_{s}$. If the clock is jittered, its rising edges occur at time instants $T_{s}(n+j(n))$, where $\mathrm{j}(\mathrm{n})$ is a stochastic dimensionless sequence, which we'll call jitter. For the analysis in this manuscript, we focus on the spectrum of this jitter, which can be very complicated. In a PLL, several noise sources exist, generating, among others, white noise and $1 / f$ noise. These contribute to the clock jitter, after they are shaped by different filters, depending on where they are injected in the loop. Sometimes also high frequency spurs ([22, p. 17]) are present in $j(n)$. As was done in [6], where CTSDMs were considered, we will model the jitter, $j$, as white Gaussian noise, $j_{0}$, which has been filtered by a low pass filter, $L P_{j}(z)$. This is depicted at Fig 8. By a suitable choice of the filter $L P_{j}$, we can shape the spectrum of $j(n)$ to mimic a broad range of real world jitter spectra. This allows us to investigate the influence of these jitter spectra on the modulator in an analytical way.

In this study, we want to separate the effect of the jitter spectral shape from the influence of the jitter power. Therefore we will assume that the filter $L P_{j}$ is normalized, as was also done in [6]:

$$
\frac{1}{2 \pi} \int_{-\pi}^{\pi}\left|L P_{j}\left(e^{\omega I}\right)\right|^{2} d \omega=1
$$

This normalization ensures that the filter only represents the relative distribution of the jitter's power over frequency, and has no influence on the total jitter power, $\sigma_{j}^{2}$.

\section{B. Jitter-caused errors}

It is well known that a jittered clock degrades the performance of a continuous time sigma delta modulator ([2,5,6]). In the following, we will apply the analysis of [2] on SOSDMs.

As indicated on Fig. 1, small stochastic variations on the clock edges introduce noise at two places in the system. Firstly the jitter affects the sampling, and the associated error is called "missampling". This is a small error, and its effect will be shaped by the loop filter of the system, similarly to the suppression of the quantization noise. Hence it can be neglected in all practical cases $([2,4])$.

The second jitter-caused error is introduced at the DAC. The DAC drives the system with two-level NRZ pulses. Every time the output signal of the system changes, the ideal DAC output signal switches. This normally only happens at time instants that are multiples of the sampling period. But because of jitter, these switching times are corrupted, which leads to small errors on the pulse widths, as is depicted on Fig. 7. Errors in the pulse widths are not suppressed by the loop filter. Therefore this noise source is dominant over the missampling, so we will restrict our analysis to this error-contribution.

Calculating the effect of these varying pulse widths at the DAC on the modulators output signal is not straightforward. It is far more easy to consider sampled additive error signals. We will therefore not model the jitter induced error signal at the DAC as a pulse with a fixed level and a stochastically varying width, but as an equivalent pulse with a fixed width and a varying level (see Fig. 7). If both types of error pulses have the same area, their low frequency, in-band content will remain the same. Hence, accurate results will be obtained for the system's signal to noise ratio by considering these equivalent error pulses. 


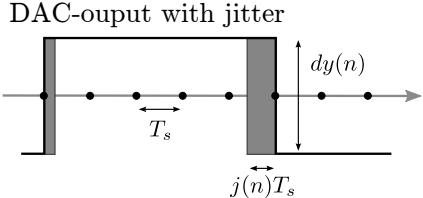

Equivalent error pulses

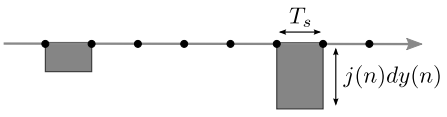

Fig. 7. Model of jitter-caused pulse-width errors at the DAC

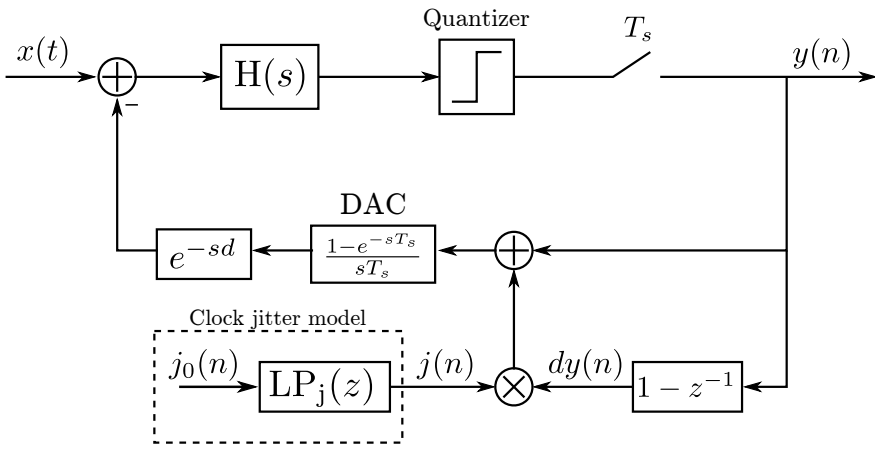

Fig. 8. Jitter model based on [2] for SOSDM

Fig. 8 shows the corresponding model for the effect of jitter. Note that the (dimensionless) jitter noise is multiplied with the differentiated output signal, $d y$, indicating that errors are only introduced when the modulator's output signal changes. Because of this multiplication, this model is not linear, and to find an exact expression for the output signal, a non-linear system equation needs to be solved. Therefore, we will use perturbation theory ([23, p. 319]) to approximate the output signal of the modulator. This means that we assume that the effect of jitter on the system is small, and represent the output signal of the modulator as the superposition of $y_{0}$, the output signal of the system without jitter, and a term $y_{j}$, which is a small disturbance on the output signal caused by the jitter:

$$
y(n)=y_{0}(n)+y_{j}(n)
$$

We will call $y_{j}(n)$ the jitter noise of the system. Coupling Eq. (20) with the model of figure 8 we find:

$$
y_{j}(n)=(d y(n) j(n)) \circledast g(n)
$$

'®' represents the convolution operation and $d y$ is to be interpreted as the discrete differentiation operator, $d$, operating on $y . g(n)$ is the impulse response of the filter $G(z)$ :

$$
G(z)=\frac{L G(z)}{1+L G(z)} \approx 1
$$

where $L G(z)$ is the z-domain loop gain of the modulator given by Eq. (18). By approximating the filter $G(z)$ as a unity operation, and neglecting the second order jitter term, $d y_{j}(n) j(n)$, we finally obtain:

$$
\begin{gathered}
y_{j}(n) \approx d y_{0}(n) j(n) \\
\text { where } d y_{0}(n)=y_{0}(n)-y_{0}(n-1)
\end{gathered}
$$

For a CTSDM with high loop gain and a NRZ DAC pulse, neglecting the second order jitter term was shown to be very accurate by the theory in [5], and simulation results seem to indicate that it remains accurate for SOSDMs. To calculate $y_{0}$, the output signal of the modulator in the absence of jitter, we will use Eq. (15) derived in the previous section.

\section{EXPRESSIONS FOR THE JITTER NOISE POWER}

\section{A. General expressions}

In order to study the jitter noise $y_{j}$, we will calculate its power spectral density $S_{y j}$. In the rest of this discussion we will use the notation $S_{z}$ to represent the power spectral density (PSD) of the signal $z$. To calculate $S_{y j}$, we assume that the jitter, $j(n)$, and the differentiated jitterless output signal, $d y_{0}(n)$, are uncorrelated. Starting from Eq. (23), we find:

$$
\begin{aligned}
S_{y j}(\omega) & =S_{d y_{0}} \circledast S_{j} \\
& =\frac{\sigma_{j}^{2}}{2 \pi} \int_{-\pi}^{\pi} S_{y_{0}}(\theta)\left|1-e^{-I \theta}\right|^{2}\left|L P_{j}\left(e^{I(\omega-\theta)}\right)\right|^{2} d \theta
\end{aligned}
$$

Eq. (25) again illustrates that the differentiated jitterless output signal of the system, $d y_{0}$, is modulated by the jitter. As was shown in section II, the jitterless output signal consists of three distinct components: the oscillation, the input signal and the quantization noise. If we assume that these components are uncorrelated, we can rewrite Eq. (25) as follows:

$$
S_{y j}(\omega)=\left(S_{d y_{0}, x}+S_{d y_{0}, c}+S_{d y_{0}, q}\right) \circledast S_{j}
$$

From this, it is evident that the jitter noise in the output signal can be split up in three components. The first component, $y_{j, x}$, is related to the input signal, and has a PSD equal to $S_{y j, x}$. The second component, $y_{j, c}$, is related to the oscillation signal, and its PSD is $S_{y j, c}$. The last component, $y_{j, q}$, is related to the quantization noise. Its PSD is given by $S_{y j, q}$.

$$
\begin{aligned}
& S_{y j, x}(\omega)=S_{d y_{0}, x} \circledast S_{j} \\
& S_{y j, c}(\omega)=S_{d y_{0}, c} \circledast S_{j} \\
& S_{y j, q}(\omega)=S_{d y_{0}, q} \circledast S_{j}
\end{aligned}
$$

In other words, the clock jitter modulates the three differentiated signal components in the loop, resulting in three components in the jitter noise of the system.

\section{B. Separate jitter contributions}

Next we try to evaluate and simplify Eqs. (27-29), using the results of section II.

1) Input signal related jitter noise: The PSD of the jitter noise related to the sinusoidal input signal is quite easily derived:

$$
\begin{aligned}
S_{y j, x}(\omega) & =\sigma_{j}^{2} \frac{A_{x}^{2}}{4}\left|\left(1-e^{-I \omega_{x} T_{s}}\right)\right|^{2} \\
& \left(\left|L P_{j}\left(e^{I\left(\omega-\omega_{x}\right) T_{s}}\right)\right|^{2}+\left|L P_{j}\left(e^{I\left(\omega+\omega_{x}\right) T_{s}}\right)\right|^{2}\right)
\end{aligned}
$$

The input signal will shift the low pass jitter spectrum to the input signal frequency, creating a skirt around the input signal component in the output signal of the modulator. It is important to note that this input signal related jitter noise 
component is quite fundamentally linked to sample and hold mechanisms, and it is not specifically caused by the SOSDM architecture. Indeed, the PSD of the output signal of a simple open loop sample and hold circuit without quantization would also contain such a skirt around the input signal component if it was sampled with the same jittered sampling clock ([3]). This input signal related jitter noise is simply phase noise caused by sampling on varying instances. In the following treatment, this noise will be handled as if it were additive noise. However, this can be misleading, since several systems are quite robust against this phase noise, and algorithms exist that mitigate its effect, under certain circumstances (e.g. [24]).

2) Oscillation related jitter noise: For the PSD of the oscillation related jitter noise, an exact expression is also readily derived using Eq. (28):

$$
\begin{aligned}
& S_{y j, c}(\omega)=\sigma_{j}^{2} \frac{A_{c}^{2}}{4}\left|\left(1-e^{-I \omega_{c} T_{s}}\right)\right|^{2} \\
& \left(\left|L P_{j}\left(e^{I\left(\omega-\omega_{c}\right) T_{s}}\right)\right|^{2}+\left|L P_{j}\left(e^{I\left(\omega+\omega_{c}\right) T_{s}}\right)\right|^{2}\right)
\end{aligned}
$$

Eq. (31) shows that the oscillation will shift the jitter spectrum components at the oscillation frequency to base band. We can approximate Eq. (31) as a constant for low frequencies. If the modulator has a small bandwidth and a high oscillation frequency, this approximation is fairly accurate, and leads to the following simple expression for the in-band oscillation related jitter noise power:

$$
\begin{aligned}
P_{y j, c} & \approx \frac{1}{O S R} S_{y j, c}(0), \quad \text { if } \frac{\pi f_{s}}{O S R} \ll \omega_{c} \\
& =\frac{1}{O S R} \sigma_{j}^{2} \frac{A_{c}^{2}}{2}\left|1-e^{-I \omega_{c} T_{s}}\right|^{2}\left|L P_{j}\left(e^{I \omega_{c} T_{s}}\right)\right|^{2}
\end{aligned}
$$

The condition of Eq. (32) is a reasonable one, since in a good design the carrier and its spurs should lie far away from the signal band. Eq. (33) shows that the oscillation related jitter noise does not directly depend on the modulator's loop filter. For a given clock, the jitter noise mainly depends on the oscillation frequency. This frequency will often be chosen early in the design process to obtain a certain power consumption or quantization noise suppression. Also the oscillation amplitude, $A_{c}$, is quite constant. Hence, there is little control on the oscillation related jitter noise while designing the modulator. There is however some control in choosing the sampling clock. Eq. 33 shows that choosing a clock whose jitter is small around the oscillation frequency, will reduce the oscillation related jitter noise.

3) Quantization noise related jitter noise: Expressions for quantization noise related jitter noise are more difficult to derive and depend on the exact NTF and jitter spectrum. The exact value of the quantization related jitter noise will be obtained in this paper through numerical evaluation of Eq. (29). It is however clear that the noise level at low inband frequencies is proportional to:

$$
S_{y j, q}(0) \sim \int_{-\pi}^{\pi}\left|N T F(\theta)\left(1-e^{-I \theta}\right)\right|^{2}\left|L P_{j}\left(e^{I \theta}\right)\right|^{2} d \theta
$$

Since the NTF is a high pass filter, this equation indicates that the in-band quantization noise related jitter decreases if the energy of the clock jitter is concentrated at low, in-band frequencies.

\section{First order shaped jitter}

In many PLL-based clock generation systems, the jitter spectrum can often be well approximated as a first order shaped spectrum, especially at the higher out of band frequencies where the quantization noise and the oscillation have high power ([6]). Considering a first order shaped spectrum also allows us to analytically derive some closed form expressions that can be used to understand the basic jitter mechanisms. Therefore we will offer expressions and approximations for the noise component in the modulator's output signal caused by clock jitter with a spectrum that is approximately first order shaped. To describe those first order spectra, we will assume that $L P_{j}$ has the following form ([6]):

$$
L P_{j}(z)=\frac{\sqrt{1-z_{p}^{2}}}{1-z_{p} z^{-1}}
$$

Here $L P_{j}(z)$ is the Z-transform of the sampled impulse response of the filter with transfer function $\frac{1}{1+s \tau_{j}}$, which has consequently been normalized using Eq. (19).

$$
L P_{j}(z) \sim\left\{\frac{1}{1+s \tau_{j}}\right\}^{*} \text { and } z_{p}=e^{-\frac{T_{s}}{\tau_{j}}}
$$

As in Eq. (18), $\{A(s)\}^{*}$ represents the star operator operating on a filter $A(s)$. This is equivalent to taking the Z-transform of the sampled inverse Laplace transform of $A(s)$.

1) Input signal related jitter noise: To find the in-band noise power related to the modulation of the input signal by the clock jitter, the latter's spectrum needs to be fully known. Calculations for a first order shaped jitter spectrum and a sinusoidal input signal have been performed in literature, for the case of a continuous-time sigma delta modulator. The resulting expression will be identical for a SOSDM and a CTSDM with the same DAC, and we give the result as obtained in [6]:

$$
\begin{aligned}
P_{y j, x}=\frac{4 \sigma_{j}^{2} A_{x}^{2}}{\pi} \sin ^{2} & \left(\frac{\omega_{x} T_{s}}{2}\right) \\
& \arctan \left[\frac{1+z p}{1-z p} \tan \left(\frac{\pi}{2 O S R}\right)\right]
\end{aligned}
$$

2) Oscillation related jitter noise: Eq. (33) for the in-band oscillation related jitter noise power, can further be approximated for the case of first order shaped clock jitter, if the bandwidth of this clock jitter is far lower than the oscillation frequency, $\omega_{c}$. Under those circumstances, the differentiation of the oscillation component, and the integration caused by the filter cancel out, and the following simple expression is derived:

$$
\begin{gathered}
\tau_{j} \omega_{c} \gg 1 \Rightarrow \\
P_{y j, c} \approx \frac{2 \sigma_{j}^{2} T_{s}}{\tau_{j} O S R} \frac{A_{c}^{2}}{2}
\end{gathered}
$$

Where $A_{c}$ is given by Eq. (6). 
Eq. (38) shows that the oscillation related jitter noise power is proportional to the inverse of the jitter bandwidth, for low jitter bandwidths. It is noteworthy that Eq. (38) does not depend on the oscillation frequency. For higher order shaped jitter spectra, the oscillation frequency needs to be known together with the jitter level at that frequency in order to calculate this noise power.

3) Quantization noise related jitter noise: As was previously mentioned, obtaining exact results for the quantization noise related jitter noise component in the output signal of the SOSDM, is not possible without having detailed knowledge of the noise transfer function of the modulator. This remains true for the case of first order shaped jitter spectra. We will however present an approximation, based on the assumption that the magnitude of the NTF is negligible at frequencies lower than a certain frequency $\omega_{q}$ :

$$
\left|N T F\left(e^{I \omega T_{s}}\right)\right| \approx 0 \text { for } \omega<\omega_{q}
$$

Under this assumption, the power spectral density for low frequencies of the quantization noise related jitter noise is given by:

$S_{y j, q}(0)=\frac{\sigma_{q}^{2} \sigma_{j}^{2}}{2 \pi} 2 \int_{\omega_{q} T_{s}}^{\pi}\left|N T F\left(e^{I \theta}\right)\left(1-e^{-I \theta}\right) L P_{j}\left(e^{I \theta}\right)\right|^{2} d \theta$

If the bandwidth of the clock jitter is far lower than $\omega_{q}$, the differentiation and integration by $L P_{j}$ again cancel out and Eq. (40) reduces to:

$$
\begin{gathered}
\tau_{j} \omega_{q} \gg 1 \Rightarrow \\
S_{y j, q} \approx \frac{2 \sigma_{j}^{2} T_{s}}{\tau_{j}} \frac{\sigma_{q}^{2}}{\pi} \int_{\omega_{q} T_{s}}^{\pi}\left|N T F\left(e^{I \theta}\right)\right|^{2} d \theta
\end{gathered}
$$

which leads us to an approximation for the in-band power of the quantization noise related jitter noise, $P_{y j, q}$ :

$$
P_{y j, q} \approx \frac{2 \sigma_{j}^{2} T_{s}}{\tau_{j} O S R} \sigma_{q}^{2}(N T F)_{2}
$$

$(N T F)_{2}$ is the 2-norm of the NTF. Multiplied with the quantization noise variance, $\sigma_{q}^{2}$, it represents the total quantization noise power at the output of the modulator. Eq. (43) is very similar to Eq. (38), the result for the oscillation related jitter noise. In fact it could simply be obtained from Eq. (38) by interchanging the power of the self-oscillation with the total power of the quantization noise in the output signal. Both approximations illustrate that the jitter bandwidth should be chosen as small as possible for optimal jitter performance.

\section{VALIDATION OF THE ANALYTICAL EXPRESSIONS THROUGH SIMULATION}

To illustrate the accuracy of the previous analysis, and to show the spectra of the three components of the jitter noise of a self-oscillating sigma delta modulator, a simulation was performed on modulator 1 from Table II. Fig. 9 shows the spectrum of the resulting output signal.

This simulation used a model with a quantizer and a sample and hold block that samples at stochastically varying time instances. These timing instances where calculated starting

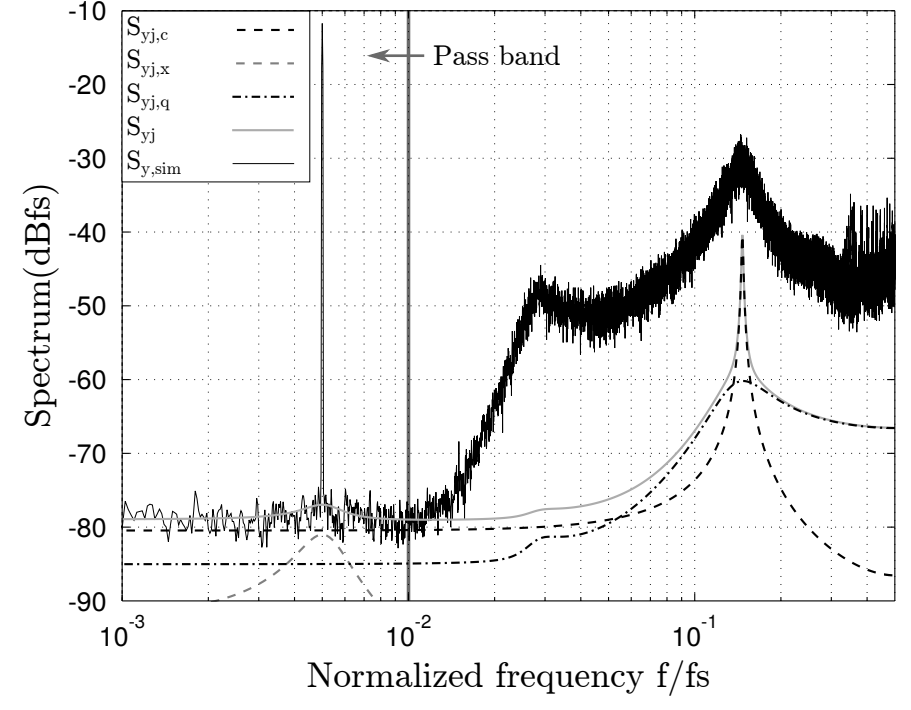

Fig. 9. Simulation results and analytical expressions for the power spectral density of the output signal and its separate jitter noise components, obtained through averaging 10 periodograms of 10000 points. The results are for modulator 1 with coefficients in Table II.

from white Gaussian noise, that was filtered by a first order filter $L P_{j}$. Its bandwidth equaled $\frac{f_{s}}{1000}$. This value was chosen to make the skirt around the input signal component visible in the output signal's spectrum. The jitter standard deviation, $\sigma_{j}$ has a value of $10 \%$, which was intentionally chosen excessively high to ensure that the quantization noise is negligible compared to the jitter noise for in-band frequencies. An analytical prediction for the PSD of the total jitter noise, $S_{y j}$, is also plotted on Fig. 9. It was obtained by numerically evaluating Eq. (26). As can be seen, it excellently matches the simulated spectrum in the low-frequency passband. At higher frequencies, the quantization noise becomes dominant over the jitter noise. Its analytical counterpart has not been plotted, as not to overload the figure.

Analytical predictions for the separate jitter noise components are also plotted. The input signal related jitter noise PSD, $S_{y j, x}$, the oscillation related jitter noise PSD, $S_{y j, c}$, and the quantization noise related jitter noise PSD, $S_{y j, q}$, were obtained by numerically evaluating Eqs. (27-29). We now shortly discuss them, starting with the self-oscillation related component. Because the jitter has a low pass spectrum, the oscillation related jitter noise is concentrated around the oscillation frequency, while it causes a noise floor in the pass band. As Eq. (33) shows, this noise floor scales with $\left|L P_{j}\left(e^{I \omega_{c}}\right)\right|^{2}$. This means that it is beneficial to have a low jitter bandwidth with preferably a steep roll-off. In practice however the roll-off may very often only be of 1 st order (see section IV-C).

Similar conclusions can be drawn with regard to the quantization related jitter noise. The reason being that the energy of the quantization noise is also concentrated at higher out of band frequencies, just like the self-oscillation's energy. So if the clock jitter spectrum is concentrated at low frequencies, only a small amount of the quantization noise will be modulated to the pass band and vice versa. The reverse can be said 
about the PSD of the input signal related jitter, $S_{y j, x}$. Its skirt falls in the pass band, and if the jitter is concentrated at low frequencies, the contribution of the input signal related jitter will be concentrated in the pass band around the input signal frequency.

To further validate the analytical expressions presented in this paper, two additional sets of simulations were performed, on the two self-oscillating sigma delta modulators whose parameters are listed in Table II. Again a sinusoidal input signal and first order shaped jitter were considered. The first set, was obtained by sweeping the bandwidth of the jitter filter, $f_{j}$, for a constant input signal amplitude of $-6 \mathrm{dBfs}$. This set of simulations was performed for severall values of the jitter standard deviation $\sigma_{j}$. The results are plotted on Fig. 10 for modulator 1, and Fig. 11 for modulator 2. On both figures, two analytical SNR predictions are also plotted. The first, labeled Analytical "exact", results from the numerical evaluation of Eq. (25), using the results of Section II for the jitterless output signal $y_{0}$. The second is labeled Analytical approx. It results from the evaluation of the approximations given by Eqs. (38) and (43), coupled with Eq. (36). Both analytical results also include the quantization noise obtained in section II.

Good correspondence is found between simulation and the "exact" analytical results. The error for low jitter bandwidths is always smaller than $1.9 \mathrm{~dB}$. For higher jitter bandwidths, the error on the "exact" analytical result increases. The reason is that approximating the oscillation as a sine becomes less accurate when the jitter bandwidth lies close to the oscillation frequency. But even for very high jitter bandwidths, the error remains smaller than $2.5 \mathrm{~dB}$. The analytical approximation is also quite accurate. The $-10 \frac{\mathrm{dB}}{\mathrm{dec}}$ slope predicted by the $\frac{1}{\tau_{j}}$ dependence in Eqs. (38) and (43), for the oscillation and quantization noise related in-band jitter noise power, is clearly visible on Fig. 10. Only for the lowest and the highest jitter bandwidths, the SNR's slope softens. For the lowest jitter bandwidths the input signal related jitter noise becomes dominant. At the highest jitter bandwidths, the assumptions of Eqs. (37) and (41) are no longer valid, and as a result, the accuracy of the approximations made on these assumptions becomes less good.

The second set of simulation results consisted of sweeping the amplitude of the sinusoidal input signal, for a constant jitter bandwidth of $\frac{f_{s}}{1000}$, and again with $\sigma_{j}=10 \%$. These simulations were also performed on both modulators in Table II. Fig. 12 shows the simulation results and analytical predictions of the SNR of the modulator. Their difference is always smaller than $1.2 \mathrm{~dB}$, so we can safely say that once again, the simulation results confirm the validity of the analytical analysis.

\section{Clock JitTER SPURS}

Sometimes clock jitter spectra contain spurs at high out of band frequencies [21]. Because of the presence of the self-oscillation in the modulator's loop, spurs in the clock jitter spectrum at the oscillation frequency can have a severe effect on the SOSDM's performance. For such a clock jitter spectrum, the carrier's energy is modulated to baseband by

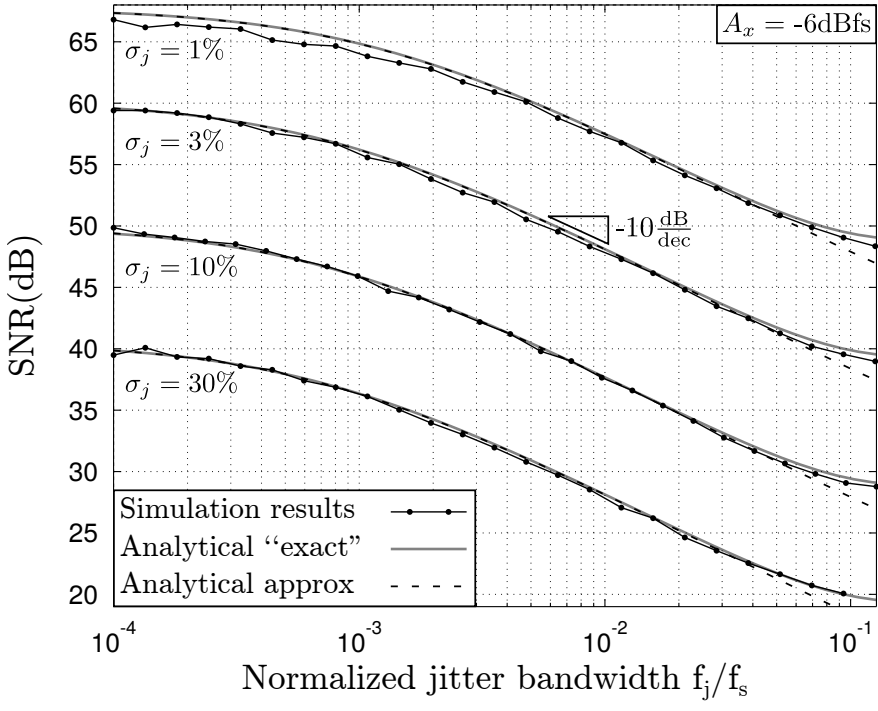

Fig. 10. Simulated SNR for Modulator 1 (see Table II) vs. the jitter bandwidth for several jitter variance values. The input signal was a $-6 \mathrm{dBfs}$ sine wave. The clock jitter had a first order shaped spectrum. The analytical results and the approximations derived in Section IV are also plotted.

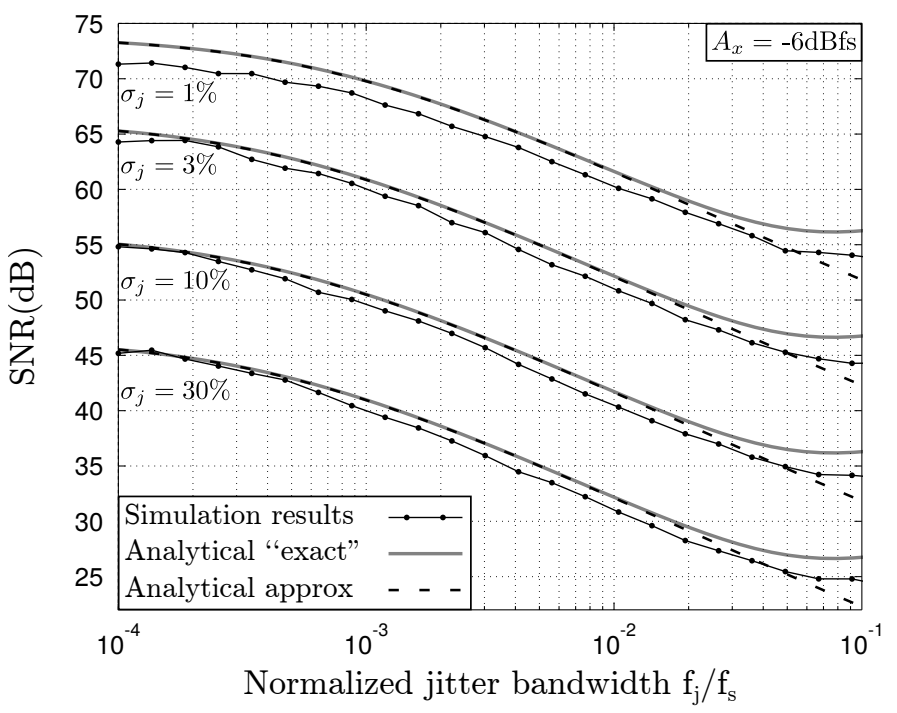

Fig. 11. Simulated SNR for Modulator 2 (see Table II) vs. the jitter bandwidth for several jitter variance values. The input signal was a $-6 \mathrm{dBfs}$ sine wave. The clock jitter had a first order shaped spectrum. The analytical results and the approximations derived in Section IV are also plotted.

the spurious jitter. Since the self-oscillation's energy is confined to a relatively limited frequency band, this modulation will generate jitter noise with high in-band energy, severely degrading the modulator's SNR.

In this paper, we approximated the self-oscillation as a sine. Unfortunately, using this approximation for the case of spurs in the clock jitter spectrum, would lead to results with bad accuracy. The reason for this is that, using this model, either all or none of the oscillation's energy would be modulated in-band, while in reality, always a part of the oscillation's energy would be modulated to baseband. Finding a better approximation for the carrier falls out of the scope of this paper, but to illustrate the mechanism, Fig. 13 shows 


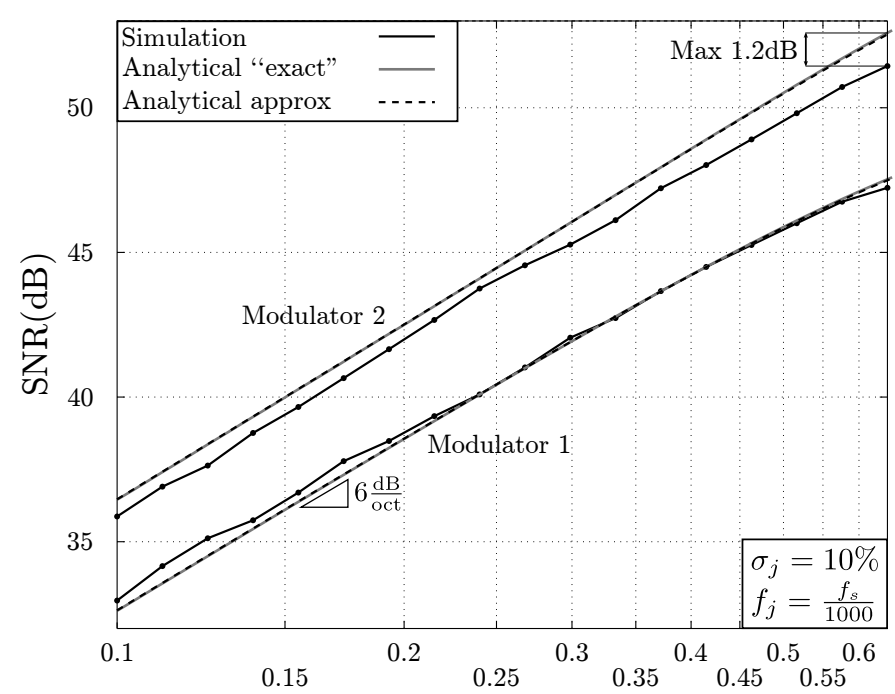

Input signal amplitude $\mathrm{A}_{\mathrm{x}}$ relative to full scale

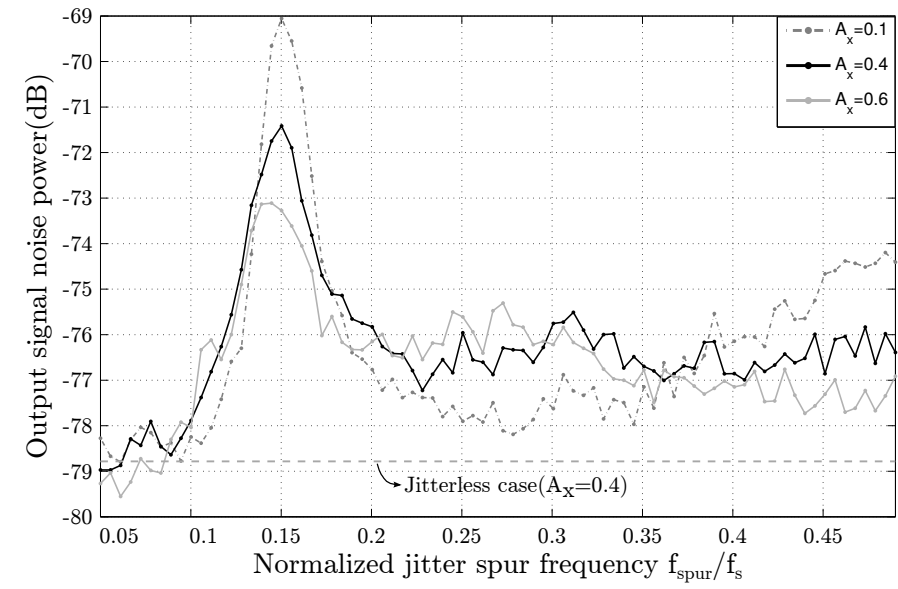

Fig. 13. Simulation of a SOSDM (Modulator 1 in Table II) whose clock jitter is a sine with frequency $f_{\text {spur }}$. The resulting noise power is plotted versus $f_{\text {spur }}$, for different input signal amplitudes, $A_{x}$.

Based on these expressions, we can conclude that the jitter noise performance depends heavily on the jitter bandwidth, which should be as low as possible, such that the jitter is concentrated at low frequency. This leads to lower inband quantization and oscillation related jitter noise than a clock whose jitter has a significant amount of energy at high frequencies. The in-band input signal related jitter noise does increase with lower jitter bandwidths, but many practical systems are unaffected by such a noise component.

For the important case of first order shaped clock jitter, very simple, but accurate, approximations where obtained for the oscillation and the quantization noise related in-band jitter noise power. It was shown that if the oscillation frequency is much higher than the jitter bandwidth, the oscillation related jitter in-band noise power does not depend on the loop filter or the oscillation frequency. It was also shown that for first order shaped jitter spectra with a low jitter bandwidth, the amount of in-band quantization noise related jitter noise is proportional to the 2-norm of the noise transfer function.

In the analysis offered by this paper, the oscillation was approximated as a single tone without sidebands. This proved accurate for most of the considered jitter spectra. However, the proposed model does not accurately describe the jitter performance in one potentially very dangerous situation. When there are spurs in the jitter spectrum near the oscillation frequency, a large amount of noise can be modulated to the system's passband, and designers should take care, since this is not accurately modeled by the proposed model.

\section{APPENDIX}

In this appendix we will derive an average oscillation amplitude, and we will give an expression for $f_{0}$, that was derived in [15]. Like in section II, we neglect the sampling, so that the resulting system represents an asynchronous sigma delta modulator. We also consider a slowly varying, sinusoidal input signal $x$, which remains approximately constant over several oscillation periods. The Fourier series of the square wave at the modulator's output, which has a slowly varying these jitter caused noise components. These expressions show excellent correspondence with system level simulation results.

\section{was shown that if the clock signal of a SOSDM con} jitter, the three components in the SOSDM's output signal modulated, leading to three jitter noise components. Analytical expressions were derived for the power spectral density of 
duty cycle $\alpha$ and period $T_{c}=\frac{2 \pi}{\omega_{c}}$, is given by [15]:

$$
(2 \alpha-1)+4 \sum_{n=1}^{\infty} \frac{\sin (n \pi \alpha)}{n \pi} \cos \left(n \omega_{c} t\right)
$$

The first term $(2 \alpha-1)$ contains the low frequency content of the output signal. For a slowly varying input signal, and an integrating loop filter, it is approximately equal to $x$.

$$
\alpha=\frac{1+x}{2}
$$

The other terms in Eq. (44) are the oscillation and its harmonics. If we neglect these harmonics and only consider the fundamental tone, the result is not a sine wave, because $\alpha$ and $\omega_{c}$ vary in time, resulting in a broadening of the oscillation's spectrum. We will however represent the oscillation as a single tone, and to this end, we calculate an average oscillation amplitude, $A_{c}$.

\section{Oscillation amplitude}

We define $A_{c}$ as that amplitude that results in a sine wave with the same power as the first harmonic of the pulse width modulated square wave output signal of the SOSDM. Although this is an approximation, it gives us accurate results for the in-band jitter noise contribution of the oscillation. From Eq. (44) the time-varying amplitude of the fundamental tone is

$$
A_{1}=\frac{4}{\pi} \sin (\pi \alpha)
$$

By calculating the rms value of $A_{1}$ we obtain:

$$
\begin{aligned}
A_{c} & =\sqrt{\left(\frac{4}{\pi}\right)^{2} \frac{1}{2 \pi} \int_{-\pi}^{\pi} \sin ^{2}\left(\frac{\pi}{2}\left(1+A_{x} \sin (\phi)\right)\right) d \phi} \\
& =\frac{4}{\pi} \frac{1}{\sqrt{2}} \sqrt{1+J_{0}\left(A_{x} \pi\right)}
\end{aligned}
$$

Where $J_{0}$ is the zeroth order Bessel function of the first kind, $A_{x}$ is the amplitude of the input signal, and $\omega_{x}$ is its angular frequency.

\section{Oscillation frequency}

In section II, an expression was given for $f_{0}$, the zero-input oscillation frequency, for a first order ASDM. In [15] however, an expression for $f_{0}$ is derived for a general order ASDM, under certain assumptions. Firstly, the order of the numerator of the (continuous-time) loop filter should be exactly one order higher than the denominator's order. Secondly the poles and zeros of the filter should be much lower in frequency than the oscillation. Also the real and imaginary parts of the loop filter should comply with the following conditions:

$$
\operatorname{Re}\{H(j \omega)\} \approx \frac{1}{\left(\tau_{r} \omega\right)^{2}}, \operatorname{Im}\{H(j \omega)\} \approx \frac{1}{\tau_{i} \omega} \text { for } \omega \gg \omega_{c}
$$

Here, $\omega_{c}$ represents the average angular oscillation frequency of the modulator. Lastly the expression was derived assuming a slowly varying, almost constant input signal. Under these conditions, the zero-input oscillation frequency is given by:

$$
f_{0}=\frac{\left(\tau_{i} d_{t}-\tau_{r}^{2}\right)}{2 d_{t}\left(\tau_{i} d_{t}-2 \tau_{r}^{2}\right)}
$$

$d_{t}$ represents the total loop delay, as given by Eq. (1).

\section{REFERENCES}

[1] K. Reddy and S. Pavan, "Fundamental limitations of continuous-time delta-sigma modulators due to clock jitter," IEEE Trans. Circuits Syst.I, vol. 54, no. 10, pp. 2184-2194, Oct. 2007.

[2] L. Hernandez, A. Wiesbauer, S. Paton, and A. Di Giandomenico, "Modelling and optimization of low pass continuous-time sigma-delta modulators for clock jitter noise reduction," in Proc. IEEE Int. Symp. Circuits and Syst. (ISCAS), 2004, pp. 1072-1075.

[3] J. A. Cherry and W. M. Snelgrove, "Clock jitter and quantizer metastability in continuous-time delta-sigma modulators," IEEE Trans. Circuits Syst.-II, vol. 46, no. 6, pp. 661-676, 1999.

[4] H. Tao, L. Toth, and J. Khoury, "Analysis of timing jitter in bandpass sigma-delta modulators," Circuits and Systems II: Analog and Digital Signal Processing, IEEE Transactions on, vol. 46, no. 8, pp. 991-1001, Aug 1999.

[5] Edward, A and Silva-Martinez, J., "General Analysis of Feedback DAC's Clock Jitter in Continuous-Time Sigma-Delta Modulators," IEEE Trans. Circuits Syst.-II: Express Briefs, vol. 61, no. 7, pp. 506-510, July 2014.

[6] Y.-S. Chang, C.-L. L. Lin, W.-S. E. Wang, C.-C. Lee, and C.-Y. Shih, "An analytical approach for quantifying clock jitter effects in continuous-time sigma-delta modulators," IEEE Trans. Circuits Syst.-I, vol. 53, no. 9, pp. 1861-1868, Sept. 2006.

[7] B. De Vuyst and P. Rombouts, "A 5-MHz 11-Bit Self-Oscillating Sigma Delta Modulator With a Delay-Based Phase Shifter in $0.025 \mathrm{~mm}^{2}$," IEEE J. Solid-State Circuits, vol. 46, no. 8, pp. 1919-1927, Aug. 2011.

[8] L. Hernandez and E. Prefasi, "Analog-to-digital conversion using noise shaping and time encoding," IEEE Trans. Circuits Syst.-I, vol. 55, no. 7, pp. 2026-2037, aug. 2008.

[9] L. Hernandez, E. Prefasi, E. Pun, and S. Paton, "A 1.2-MHz 10-bit Continuous-Time Sigma-Delta ADC Using a Time Encoding Quantizer," IEEE Trans. Circuits Syst.-II, vol. 56, no. 1, pp. 16-20, 2009.

[10] E. Prefasi, L. Hernandez, S. Paton, A. Wiesbauer, R. Gaggl, and E. Pun, "A $0.1 \mathrm{~mm}^{2}$, Wide Bandwidth Continuous-Time Sigma Delta ADC Based on a Time Encoding Quantizer in $0.13 \mu \mathrm{m}$ CMOS," IEEE J. Solid-State Circuits, vol. 44, no. 10, pp. 2745-2754, oct 2009.

[11] S. Ouzounov, H. Hegt, and A. van Roermund, "Sigma-delta modulators operating at a limit cycle," IEEE Trans. Circuits Syst.-II, vol. 53, no. 5, pp. 399-403, 2006.

[12] P. Woestyn, P. Rombouts, X. Xing, and G. Gielen, "A selectablebandwidth $3.5 \mathrm{~mW}, 0.03 \mathrm{~mm}^{2}$ self-oscillating Sigma Delta modulator with $71 \mathrm{~dB}$ dynamic range at $5 \mathrm{MHz}$ and $65 \mathrm{~dB}$ at $10 \mathrm{MHz}$ bandwidth," Analog Integr. Circuits and Signal Process., vol. 72, no. 1, pp. 55-63, Jul. 2012.

[13] E. Prefasi, S. Paton, and L. Hernandez, “A 7 mW 20 MHz BW TimeEncoding Oversampling Converter Implemented in a $0.08 \mathrm{~mm}(2) 65$ nm CMOS Circuit," IEEE J. Solid-State Circuits, vol. 46, no. 7, SI, pp. 1562-1574, Jul. 2011.

[14] A. Gelb and W. E. Vander Velde, Multiple-Input Describing Functions and Nonlinear System Design. McGraw-Hill, 1968.

[15] A. Babaie-Fishani, M. De Bock, and P. Rombouts, "Analyzing Distortion in ASDMs with Loop Delay," in Proc. IEEE Int. Symp. Circuits and Syst. (ISCAS), 2014, pp. 77-80.

[16] A. Babaie-Fishani, B. Van Keymeulen, and P. Rombouts, "Analytical Expressions for the Distortion of Asynchronous Sigma-Delta Modulators," IEEE Trans. Circuits Syst.-II: Express Briefs, vol. 60, no. 8, pp. 472-476, 2013.

[17] E. Roza, "Analog-to-digital conversion via duty-cycle modulation," IEEE Trans. Circuits Syst.-II, vol. 44, no. 11, pp. 907-914, nov 1997.

[18] J. Daniels, W. Dehaene, M. Steyaert, and A. Wiesbauer, "A/d conversion using an asynchronous delta-sigma modulator and a time-to-digital converter," in Proc. IEEE Int. Symp. Circuits and Syst. (ISCAS), may 2008, pp. $1648-1651$.

[19] K. Nielsen, "A review and comparison of pulse-width modulation (pwm) methods for analog and digital input switching power amplifiers," in Audio Engineering Society Convention 102. Audio Engineering Society, 1997. 
[20] J. De Maeyer, J. Raman, P. Rombouts, and L. Weyten, "Controlled behaviour of STF in CT Sigma Delta modulators," Electron. Lett., vol. 41, no. 16, pp. 896-897, AUG 42005.

[21] T. Riley, M. Copeland, and T. Kwasniewski, "Delta-sigma modulation in fractional-n frequency synthesis," Solid-State Circuits, IEEE Journal of, vol. 28, no. 5, pp. 553-559, May 1993.

[22] K. Shu and E. Sánchez-Sinencio, CMOS PLL Synthesizers: Analysis and Design: Analysis and Design. Springer, 2006, vol. 783.

[23] C. M. Bender and S. A. Orszag, Advanced mathematical methods for scientists and engineers I: Asymptotic methods and perturbation theory. Springer, 1999, vol. 1.

[24] S. Wu and Y. Bar-Ness, "A phase noise suppression algorithm for ofdmbased wlans," Communications Letters, IEEE, vol. 6, no. 12, pp. 535537, Dec 2002.

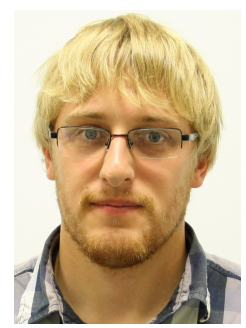

Dries Vercaemer was born in Gent, Belgium in 1990. He obtained the Ir. degree in electronics from Ghent University, Belgium in 2013. Since then he has been with the Electronics and Information Systems Department of Ghent University, where he is currently working toward a Ph.D. degree. His technical interests are analog circuit design, mainly focusing on analog digital converters.

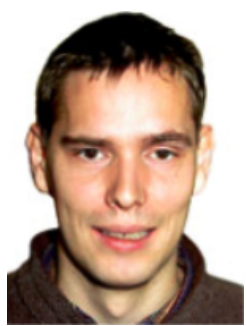

Pieter Rombouts was born in Leuven, Belgium, in 1971. He obtained the Ir. degree in applied physics and the Dr. degree in electronics from Ghent University, Belgium, in 1994 and 2000, respectively. Since 1994 he has been with the Electronics and Information Systems Department of Ghent University, where he is currently Professor. In 2002 he was a visiting professor at the University Carlos III, Madrid, Spain. He currently serves as an Associate Editor for IEEE Trans. Circuits and Syst.-Part II. His technical interests are signal processing, circuits and systems theory, and analog circuit design. 